\title{
Detection and Characterization of Human Teeth Caries Using 2D Correlation Raman Spectroscopy
}

\author{
El-Sharkawyi Y.H. ${ }^{1 *}$
}

\begin{abstract}
Background: Carious lesions are formed by a complex process of chemical interaction between dental enamel and its environment. They can cause cavities and pain, and are expensive to fix. It is hard to characterize in vivo as a result of environment factors and remineralization by ions in the oral cavity.

Objectives: The development of a technique that gives early diagnosis which is non-invasive, is of crucial importance for publichealth. Raman spectroscopy is a technique that can fulfil these requirements. The main goal of this work was to use Raman spectroscopy to differentiate between normal and carious human teethinvivo. The samples used in this study were collected by traditional human teeth.
\end{abstract}

Material and Method: An in vivo Raman spectroscopy system andspecialized fiber optic probe has been designed to obtain spectra from tissue. Theseprobes are filtered to reduce the background signal from the fiber optics and the collection fiberutilizes beam steering to optimize the collection effectiv.

Results: In order to detect any demineralization and carious versus sound pit and fissure enamel, the spectral data sets are analyzed by the proposed scheme to demonstrate the utility of generalized 2D correlation spectra. Potential applications of this 2D correlation approach are then explored. The Raman spectra in the normal tissue showed thepresence of vibrational bands in $437.87 \mathrm{~cm}^{-1}, 581.89 \mathrm{~cm}^{-1}, 953.89 \mathrm{~cm}^{-1}$ and $1054.73 \mathrm{~cm}^{-1}$ with smaller intensity than in the carious spectra. Image construction from the peak intensity produced chemical maps of apatite concentration.

Conclusion: Such two-dimensional correlation spectra emphasize spectral features not readily observable in conventional one-dimensional spectra.No correlation is observed in mode-to-mode intensity fluctuations indicating that the changes inmode intensities are completely independent. Theoretical calculations provide convincing evidence that the fluctuationsare not the result of diffusion, orientation or local electromagnetic field gradients but rather are the result of subtle variations ofthe excited-state lifetime, energy and geometry of the molecule and producing a signature response for carious detection.

\section{Keywords}

Raman Spectroscopy, 2D Correlation, Characterization of Human Teeth

\section{Introduction}

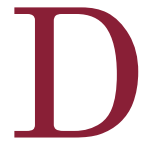

ental caries continue to be a common chronic disease among various population groups. Patient care can be improved with detection at the earliest stage. However, current techniques like visual, tactile and radiographs do not have sufficient sensitivity and specificity. The visual method cannot help in detecting the caries in its

${ }^{1}$ Department of Biomedical Engineering, Military Technical Collage, Cairo, Egypt

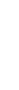

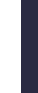

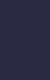

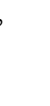


very early stage and discoloration of pits, and fissure can be misinterpreted as caries. The tactile method holds the potential to transmit cariogenic bacteria from one site to another and also may produce irreversible traumatic defects in potentially remineralizable defects.

The commonly used methods of radiographs are a two-dimensional image of a three-dimensional object. Because of this, sometimes interpretation becomes difficult. Many of these limitations have been overcome by subtraction radiography but still correct projection geometry is mandatory. The difficulty of reliably diagnosing early lesions of caries is well documented, let alone more extensive lesions [1-3]. The profession has for too long relied on good vision and clinical acumen for the clinical and radiographic diagnosis of caries. The use of new systems and techniques in the diagnosis of caries, if properly applied, can improve reliability, let alone aid in the detection of early demineralisation, which may not be clinically apparent. In addition, new diagnostic modalities allow early lesions of caries to be quantified, thereby creating the opportunity to monitor caries progression or resolution by remineralisation. Such developments reduce the reliance on subjective visual examination; although, this is still a key skill supplemented from time to time by radiographs creating opportunities for a preventative rather than the now outdated "drill-and-fill" approach to the management of caries [4-6]. This paper introduces new optical caries detection methods and a fingerprinting technique, thus materials are identified by comparing their characteristic vibrational spectra for the diagnosis of caries, mathematical formalism is proposed to obtain two-dimensional correlation spectra from any transient or time-resolved spectra having an arbitrary waveform. The 2D spectra obtained by this method can accentuate useful information often obscured in the original timeresolved spectra. This development opens up the possibility of introducing the powerful and versatile capability of 2D correlation analysis to much wider ranges of applications including complex reaction kinetics, electrochemistry and photochemistry. Further extension to other areas of spectroscopy, such as UV, Raman and ultra-fast time-resolved spectroscopy should also be quite straightforward.

The basic scheme for generating two-dimensional correlation spectra from perturbationinduced dynamic fluctuations of spectroscopic signals is similar to that already described. The general experimental approach used in 2D correlation spectroscopy is shown in Figure 1. When an external perturbation (stimulus) is applied to a system, various chemical constituents of the system are selectively excited. The excitation and subsequent relaxation processes to the equilibrium are monitored with electromagnetic probes. Typical spectral changes observed under dynamic perturbation are the variation of intensities, shift of spectral band positions and the change in the shape of peaks. The monitored fluctuation of spectral signals is then transformed into two-dimensional spectra by using a correlation method. As pointed out

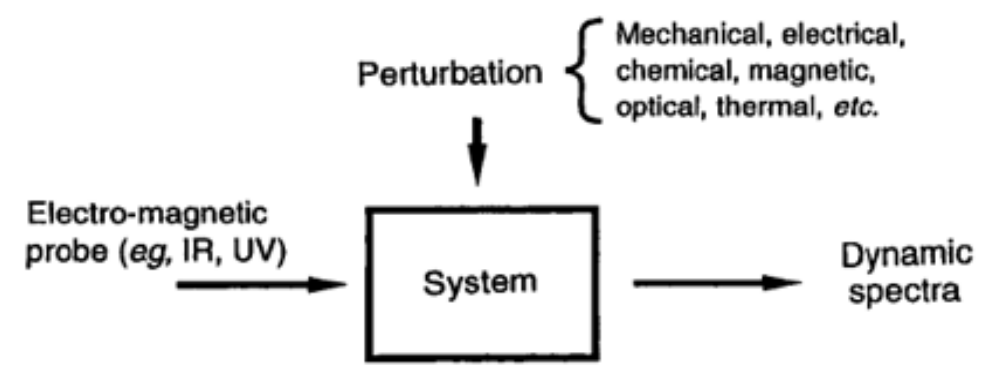

Figure 1: A general scheme for obtaining two-dimensional correlationspectra 
previously, the conceptual scheme to induce a dynamic spectrum described in Figure 1 is a very general one. It does not specify the physical nature or mechanisms through which the applied perturbation affects the system. There are, of course, many different types of external perturbations which could be used to stimulate a system of interest. For example, various molecular-level excitations may be induced by electrical, thermal, magnetic, chemical, acoustic or mechanical excitations [7-10].

Each perturbation affects the system in a unique and selective way, governed by the specific interaction mechanisms relating to the macroscopic stimuli and microscopic or molecular responses of individual system constituents. The type of physical information contained in a dynamic spectrum, therefore, is determined by the selection of perturbation method and electromagnetic probe. The interpretation of specific physical information obtained by the 2D correlation analysis, however, is beyond the scope of this paper. In order to emphasize the general applicability of the proposed 2D correlation method to a wide variety of spectroscopic problems, discussion on the subject of a specific relationship between the applied physical stimulus and dynamic spectra representing the system response is intentionally omitted. Thus, the fluctuation of spectral signals is treated on purely phenomenological grounds as a simple set of multivariate time.

\section{Materials and Methods}

A real-time NIR Raman system is shown in Figure 2. It consists of five components: light source, light delivery, Raman probe, signal delivery and signal detection (spectrometer).

The filtered light was reflected from a front surface mirror and focused onto the sample by an $\mathrm{f} / 2$ biconvex glass lens. An identical lens was used to collect the scattered light. Spectra of human teeth were obtained in vivo using Raman system with the tissue sample at the focal point of the collection lens ( $\mathrm{P}=100$ $\mathrm{mW}, \mathrm{t}=5 \mathrm{~min}$.).

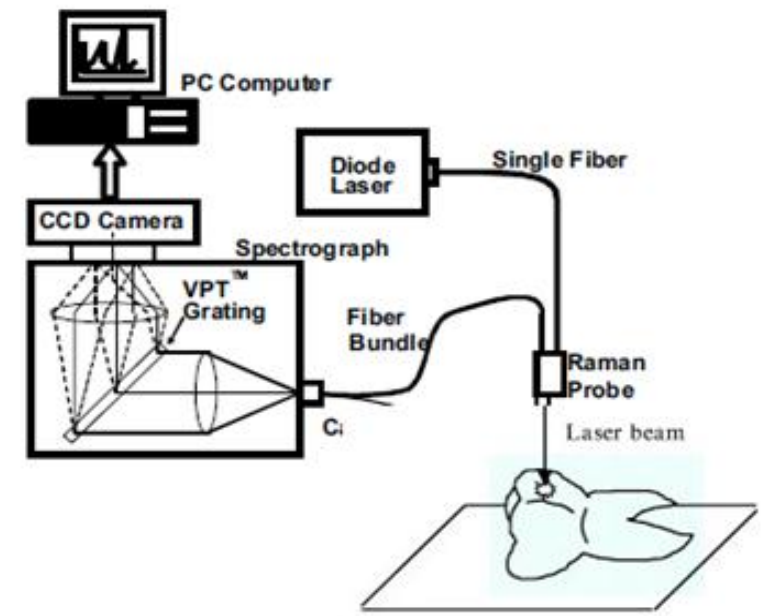

Figure 2: Block diagram of the integrated real time Raman spectrometer system for human teeth evolution and diagnosis

\section{Spectral Data Processing}

The acquired Raman spectra were pre-processed to remove artifacts caused by background fluorescence and intensity fluctuations. Using near-infrared excitation $(785 \mathrm{~nm})$ radically reduces the observance of sample auto-fluorescence to remove while-noise or other fine-scale structures from spectra. The 10-point moving average method was used in this study to smooth the spectra. Finally, all spectra were area normalized for intensity consistency at the region between 300 and $2000 \mathrm{~cm}^{-1}$. All data processing was conducted using 2D-crosscorlation algorithm.

\section{Cross-correlation Algorithms}

Synchronous two-dimensional correlation spectrum is constructed from a dynamic spectrum. A one-dimensional reference spectrum is also provided at the top and side of the $2 \mathrm{D}$ map and the deviations from the mean values of Raman intensity at different wave numbers. The correlation-coefficient plot always has a constant value of unity at the diagonal position which provides no useful spectral information. The spectral information content may be supplemented by multiplying the correlation coefficient by variances of spectral intensity 
measured at two wave numbers. This operation produces a $2 \mathrm{D}$ plot of the covariance of spectral intensities. Such a plot may be considered as a continuous form of the variancecovariance matrix of spectral intensities plotted over the entire spectral range.

Let us consider a time-dependent fluctuation of spectral intensity y $(v, t)$ observed for a period of time between - T/2 and T/2. The spectral variable $\mathrm{v}$ can be any appropriate physical variable, e.g. Raman shift. The dynamic spectrum is formally defined as $[11,12,13]$.

$y^{\prime}(v, t)=\left\{\begin{array}{c}y^{\prime}(v, t)-y^{\prime}(v), T / 2<t<-T / 2 \\ \text { otherwise }\end{array}\right.$

where $y(v)$ is the reference spectrum.

Cross-correlation Function

The two-dimensional cross-correlation function between different spectral intensities observed for a period $\mathrm{T}$ is given by $\mathrm{T}$ :

$C(\tau)=\frac{1}{T} \int_{-T / 2}^{T / 2} y^{\prime}(v 1, t) y(v 2, t+\tau) \partial t$

where $\tau$ is the correlation time and the dynamic spectral data, the synchronous $2 \mathrm{D}$ correlation spectrum can be directly computed as:

$$
\varphi(v l, v 2)=\frac{1}{T} \int_{-T / 2}^{T / 2} y^{\prime}(v 1, t) y^{\prime}(v 2, t) d t
$$

which is equivalent to the time average of the product of dynamic spectral intensities measured at two wave numbers. This feature becomes especially useful if the time-dependent spectral data are collected at irregular time intervals. Computation of the asynchronous 2D correlation spectrum, on the other hand, still requires the Fourier transform of dynamic spectrum.

\section{Disrelation Spectrum}

One of the convenient ways to circumvent the requirement of transforming spectral data into the Fourier domain is to compute a special type of asynchronous spectrum called the two-dimensional disrelation spectrum, $\Lambda\left(\mathrm{v}_{1}\right.$, $\mathrm{v}_{2}$ ). The magnitude of the disrelation spectrum is directly calculated from a set of synchro- nous correlation intensities as:

$\Lambda\left(\mathrm{v}_{1}, \mathrm{v}_{2}\right)=$ square-root $\varphi(v l, v 1) \cdot \varphi(v 2, v 2) \quad \varphi(v l, v 2)$

The use of the disrelation spectrum becomes especially attractive if the main purpose of $2 \mathrm{D}$ analysis is simply to differentiate overlapped peaks by taking advantage of the high-resolution feature of 2D spectroscopy.

In this case, only Eq. 3 needs to be evaluated since the information on temporal relations of spectral variations is not important.

\section{Results and Discussion}

Raman spectroscopy is a form of vibrational spectroscopy. It provides biochemical characterization of hydroxyapatite, the major mineral component of tooth enamel, i.e. biochemical confirmation of caries. It quantifies carious sensitivity. Difference in the morphology of sound and carious enamel rod causes shifts in Raman peaks of PO vibrations. This is due to loss of enamel crystalline orientation, i.e. induced structural changes during the carious formation process.

Figure 3 shows a worksheet designed for raw data Raman spectroscopy collected continuously from the sample (sound and carious teeth) and the signal processing used for carious detection.

So far, we have demonstrated that carious enamel can be distinguished from sound enamel at micro spectroscopic level using the intensity changes of the hydroxyapatite Raman bands. Raw data Raman spectra are not corrected for background fluorescence of sound and carious tooth are illustrated in window 1, window 2 and window 3, respectively i.e. Figure 3. Decayed teeth are classified into two classes; moderate (window 3 ) and severed carious (window 2). In windows 4, 5 and 6, the collected Raman spectra after noise reduction using 10 moving average filter. The comparison of sound enamel and carious enamel spectra is presented in windows 7 and 8 . Window 9 illustrates the signature Raman spectrum of carious position. 

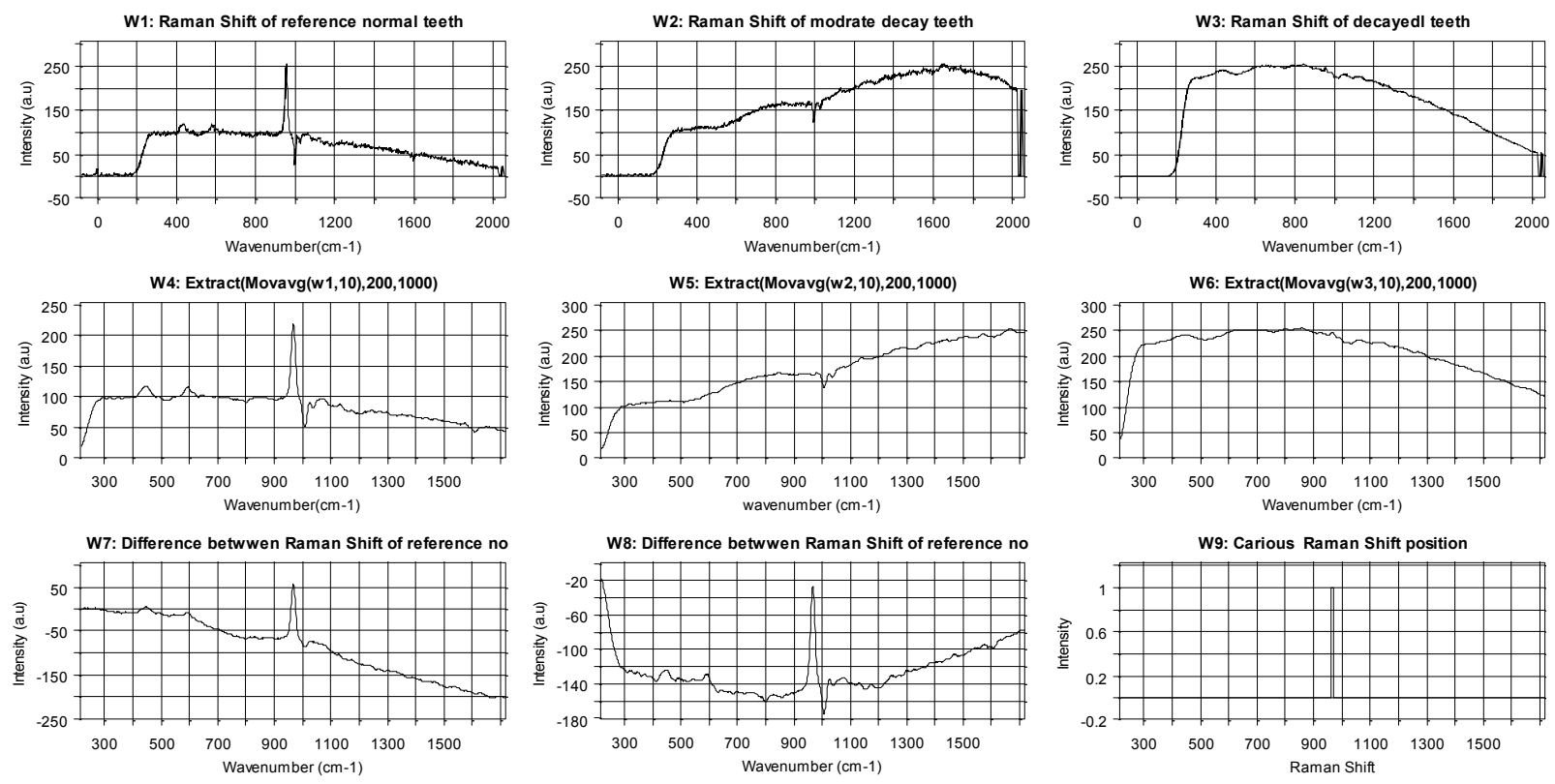

Figure 3: Raw data Raman spectra are not corrected forsound, and carious tooth are illustrated in window 1, window 2 and window 3, respectively. The decayed teeth are classified into two classes; moderate (window 3 ) and severed carious (window 2). In windows 4, 5 and 6, the collected Raman spectra after noise reduction using 10 moving average filter. The comparison of sound enamel and carious enamel spectra is presented in windows 7 and 8 . Window 9 illustrates the signature Raman spectrum of carious position.

The major hard component of teeth is dentine which is bound by cementumat, the root and a thin layer of enamel at the exposed crown. As with bone, these materials are composed of approximately $70 \%$ inorganic apatite within an organic matrix that is predominantly collagen. Smaller concentrations of protein, lipids and peptides are PO present. Enamel, the hardest tissue in human body, has the lowest concentration of organic matter and does not contain any collagen. Many researchers have studied teeth in an effort to relate composition and microstructure to their functions and mechanical properties. For example, it has been shown that susceptibility to caries is dependent on the concentration of carbonate and fluoride. As with bone, NIR Raman spectroscopy can provide information on both the mineral and the organic components of teeth.

Figure 4 illustrates the use of Raman microscope to examine the enamel-dentine junction of excised teeth. As expected, enamel spectra were dominated by apatite peaks at 437.87 (OP0 bend), 581.89 (OP0 bend),953.89 (PO stretch for $\mathrm{PO}^{3-}{ }_{4}$ ) and $1054.73 \mathrm{~cm}^{-1}$ (PO asymmetric stretch). Image construction from the intensity of $997.37 \mathrm{~cm}^{-1}$ peak produced chemical maps of apatite concentration.

Carbonate and phosphate bands identified at $1100-1000 \mathrm{~cm}^{-1}$ indicated that the concentration of carbonates increased from enamel to junction.

Visual and tactile examinations are used to detect caries so that infected tissue may be removed. This is subjective and diagnosis is hindered by the natural variation of colour and hardness of healthy teeth. Raman spectroscopy is less sensitive to colour variation and provides diagnostic information based on the biochemical and inorganic composition of the tissue.

The Raman signal was also accompanied by a broad luminescence which the authors attributed to organic matter from micro- 


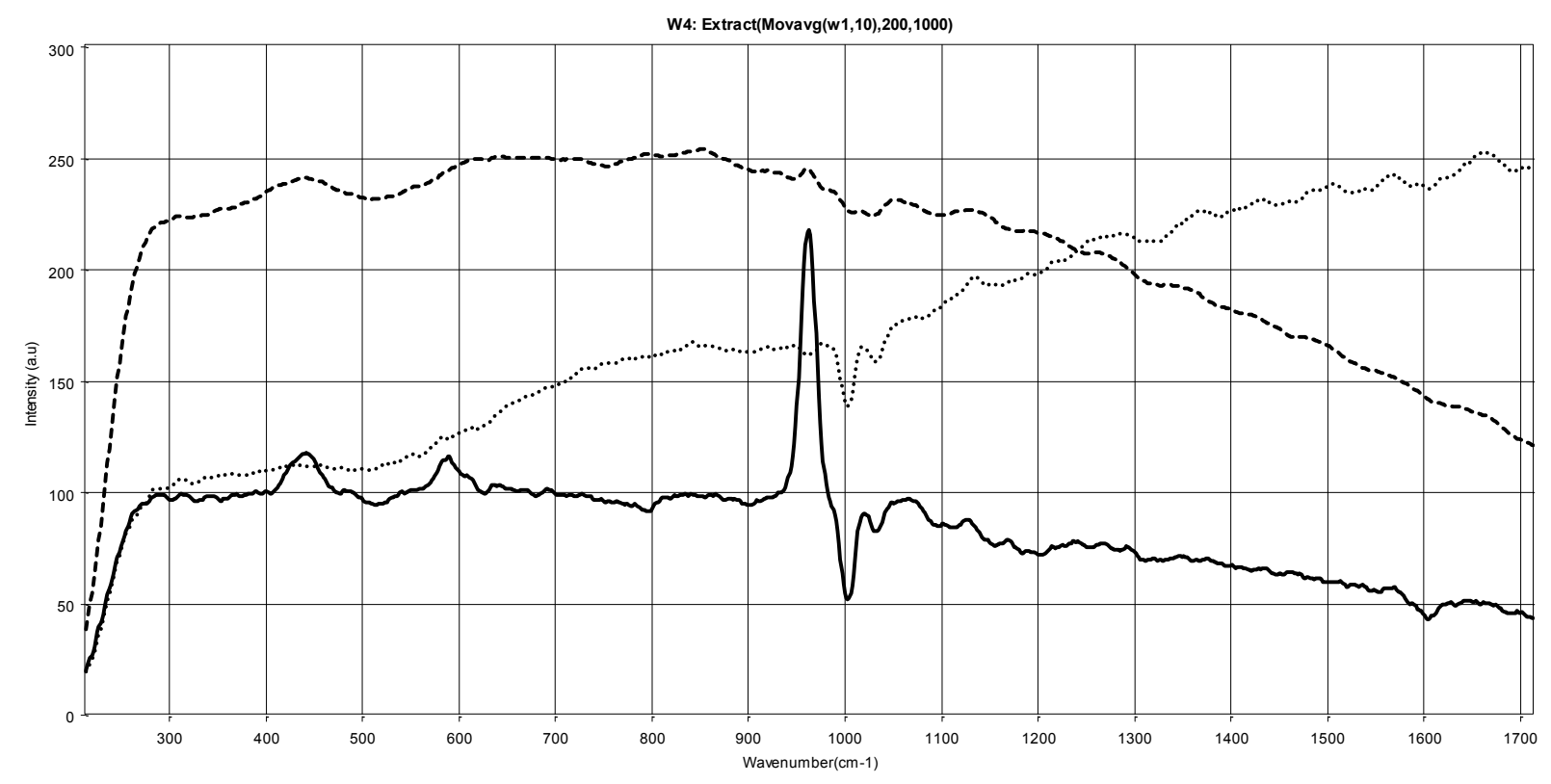

Figure 4: Spectra of healthy (bold line)and carious dentin (moderate stage (dished line) and severed stage (dot line) The maximum intensity of the chroniccariousdentin, due to the luminescent baseline is 8 times greater than the sound dentin.

biological metabolism that provided a direct measurement of bacterial infection (Figure 4). The phosphate peak at $952 \mathrm{~cm}^{-1}$ was used to quantify mineral content, while the luminescence at $1129 \mathrm{~cm}^{-1}$ was used to classify caries as shown in Figure 4. The trends of increased luminescence and decreased mineralization were also identified, to a lesser degree, in spectra of initial-stage caries. It was noted that this technique is only effective for surface examination due to the poor penetration depth of the excitation Light. The larger sampling depth obtained with the fibre probe indicates that the region beyond caries lesion and into the healthy enamel layer was possibly measured in the Raman spectra. As such, any spectral contribution from the carious lesion is gradually diminished in the overall spectrum in turn resulting in slightly decreased degree of spectral discrimination between sound and carious enamel.

Caries begin on tooth enamel, which has hydroxyapatite (HA) as its main component. When a solution containing fluoride comes in contact with $\mathrm{HA}, \mathrm{OH}$ - ions may be replaced by F- ions resulting in the formation of fluoridated apatite.

This compound is found both in enamel and dentin $[14,15]$. Raman spectroscopy shows the chemical structure of the tissue. As mentioned before, the observed enamel and dentin peaks related to the inorganic components $\sim 960 \mathrm{~cm}^{-1}$ (phosphate apatite) and $\sim 575 \mathrm{~cm}^{-1}$ (attributed to fluoridated apatite), both with greater intensity in enamel, confirm its higher mineral content.

Raman spectroscopy of carious and non-carious teeth revealed reduction of the intensity of peaks from inorganic components, but were not statistically significant. The changes in the Raman peak of phosphate between sound and carious enamel, in this study were observed as a significant reduction only for the $960 \mathrm{~cm}^{-1}$ (phosphate apatite), peak in dentin caries. Analysis of Raman caries peaks in enamel and dentin showed a statistically significant difference at Carbonate and phosphate bands identified at $1000-1200 \mathrm{~cm}^{-1}$ increased in carious, we observed a significant increase in intensity of bands at $1250-1600 \mathrm{~cm}^{-1}$ in severed carious 
status.

\section{Properties of 2D Correlation Spec- tra}

\section{Synchronous spectrum}

The synchronous 2D correlation spectrum of dynamic spectral intensity variations represents the simultaneous or coincidental changes of spectral intensities measured at $\mathrm{v}_{1}$ and $v_{2}$. Figure 3 shows a typical example of a synchronous $2 \mathrm{D}$ correlation spectrum plotted as a contour map. A synchronous spectrum is a symmetric spectrum with respect to a diagonal line corresponding to spectral coordinates $\mathrm{v}_{1}=\mathrm{v}_{2}$. Correlation peaks appear at both diagonal and off-diagonal positions. The intensity of peaks located at diagonal positions corresponds to the autocorrelation function of spectral intensity variations observed during a period $\mathrm{T}$. The diagonal peaks are therefore referred to as auto peaks.

Cross peaks located at the off-diagonal positions of a synchronous 2D spectrum represent the simultaneous changes of spectral signals at two different wave numbers. Such a synchronized change, in turn, suggests the possible existence of a coupled or related origin of the spectral intensity variation. It is often useful to construct a correlation square joining the pair of cross peaks located at opposite sides of a diagonal line drawn through the corresponding autopeaks to show the existence of coherent variation of spectra intensities at these wave numbers. While the sign of autopeaks is always positive, the sign of cross peaks can be either positive or negative. The sign of synchronous cross peaks becomes positive if the spectral intensities at corresponding wave numbers are either increasing or decreasing together as functions of time during the observation period. On the other hand, the negative sign of cross peaks indicates that one of the spectral intensities is increasing while the other is decreasing.

\section{Asynchronous Spectrum}

Figures 5 and 6 show an example of an asynchronous 2D correlation spectrum. The asynchronous spectrum of dynamic spectral intensity variations represents sequential or unsynchronized changes of spectral intensities measured at $\mathrm{v}_{1}$ and $\mathrm{v}_{2}$. The spectrum is anti symmetric with respect to the diagonal line. The asynchronous spectrum has no auto peaks consisting exclusively of cross peaks located at off-diagonal positions.

By extending lines from the spectral coordinates of cross peaks to corresponding diagonal positions, one can construct asynchronous correlation squares. An asynchronous cross peak develops only if the intensities of two

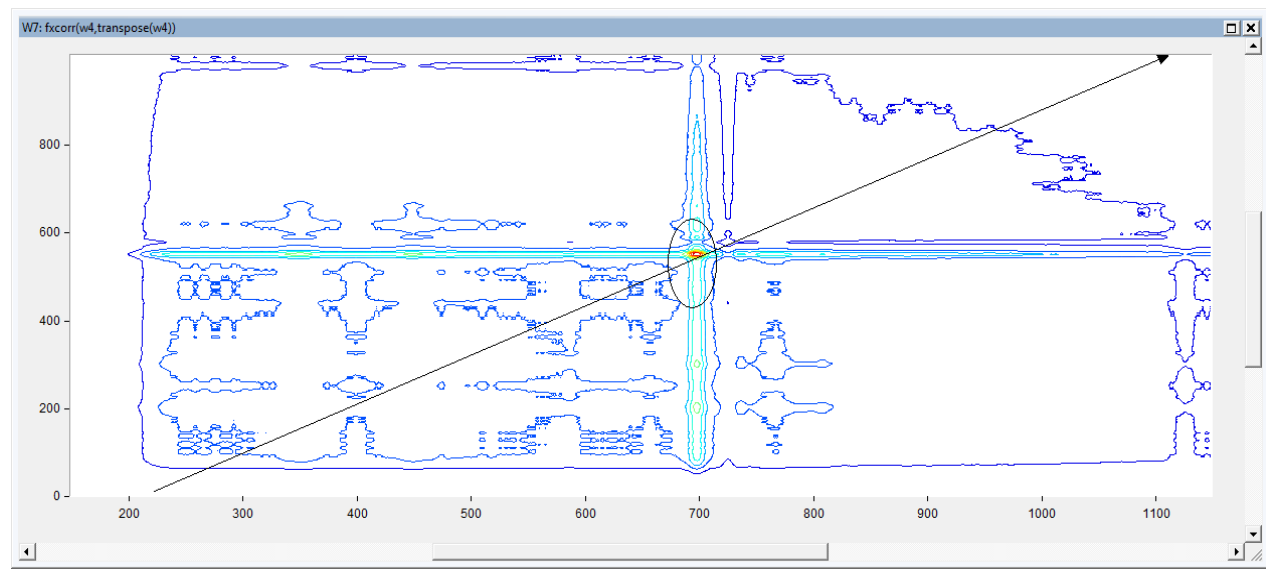

Figure 5: Shows a typical example of a synchronous 2D correlation spectrum plotted as a contour map. 


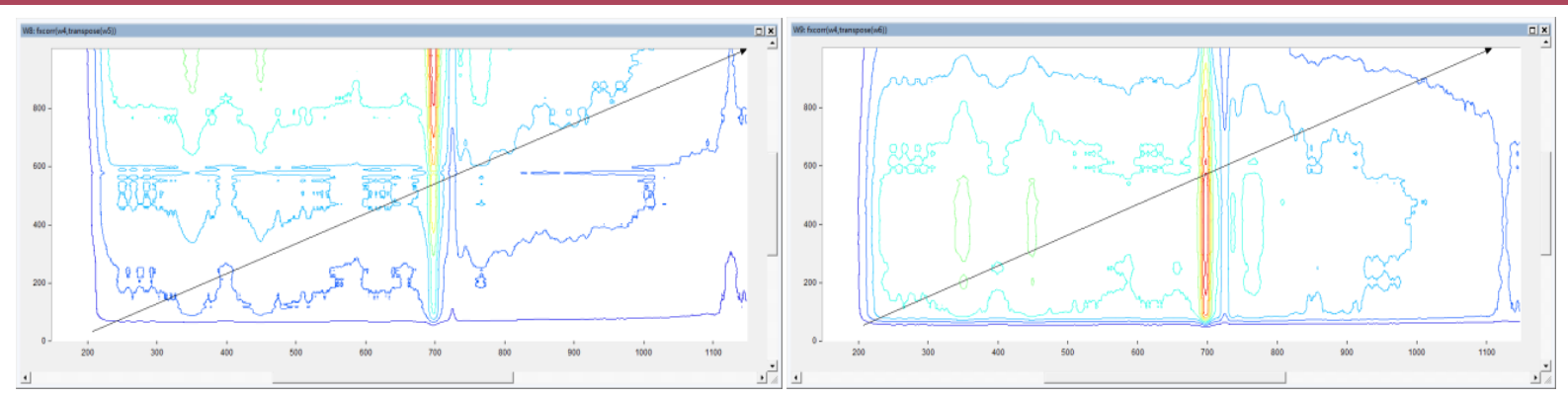

(a) Severed carious teeth

(b) Moderate carious teeth

Figure 6: Indicates the intensity changes (either an increase or a decrease) at bands Raman spectrum of carious teeth after the reference one.

dynamic spectral intensities vary out of phase (i.e., delayed or accelerated) with each other for some Fourier-frequency components of signal fluctuations.

This feature is especially useful in differentiating overlapped bands arising from different spectral origins or moieties. Thus, spectral intensity contributions from different components of a mixture, from materials in different phases or from chemical functional groups experiencing different effects due to some external field could be discriminated. Even if bands are located close to each other, as long as the temporal signatures or the patterns of timedependent variation of spectral intensities are substantially different, asynchronous cross peaks will develop among them. The appearance of asynchronous cross peaks indicates that these bands arise from different sources or functional groups in different molecular environments.

The sign of asynchronous cross peaks can be either negative (as indicated by the shaded areas) or positive. The sign of an asynchronous cross peak becomes positive if the intensity change at $v_{1}$ occurs predominantly before $v_{2}$. It becomes negative, on the other hand, if the change occurs afterwards.

\section{Disrelation spectrum}

The property of a disrelation spectrum is surprisingly similar to that of an asynchronous 2D correlation spectrum. A disrelation spec- trum is an anti-symmetric spectrum consisting of positive and negative cross peaks very much like the example spectrum shown in Figure 7. Disrelation cross peaks represent separate or independent variations of spectral intensities for all Fourier frequency components of the dynamic spectrum probed during the observation. Cross peaks develop only if the intensities of the individual spectral elements do not vary simultaneously. The temporal relationship deduced from the sign of the cross peaks is similar to that of asynchronous peaks, although disrelation peaks tend to emphasize the sequence of events occurring at higher Fourier frequencies. Thus, even though a disrelation spectrum is not quite the same as $\Lambda\left(\mathrm{v}_{1}, \mathrm{v}_{2}\right)$, it often is an excellent approximation. The major advantage of using a disrelation spectrum is that it is directly calculable from $\varphi\left(\mathrm{v}_{1}, \mathrm{v}_{2}\right)$.

In the correlation of Diagnodent readings with Raman peaks observed a negative and significant correlation only between the $\sim 575$ $\mathrm{cm}^{-1}$ and $\sim 960 \mathrm{~cm}^{-1}$ peaks and dentin caries. The lower the intensity of these inorganic components, the higher the value obtained in Diagnodent readings in dentin, which concluded that the depth of dental caries has greater influence than the mineral loss on Diagnodent readings.

In order to determine the degree of variability between the characteristic Raman spectra of each type of tissue, the 2D correlation algorithm, as discussed before, are used to detect 

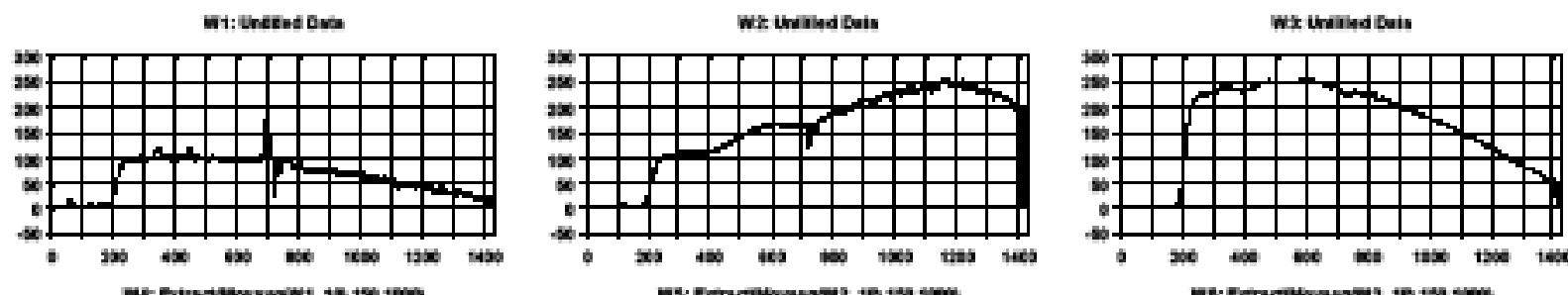

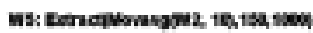
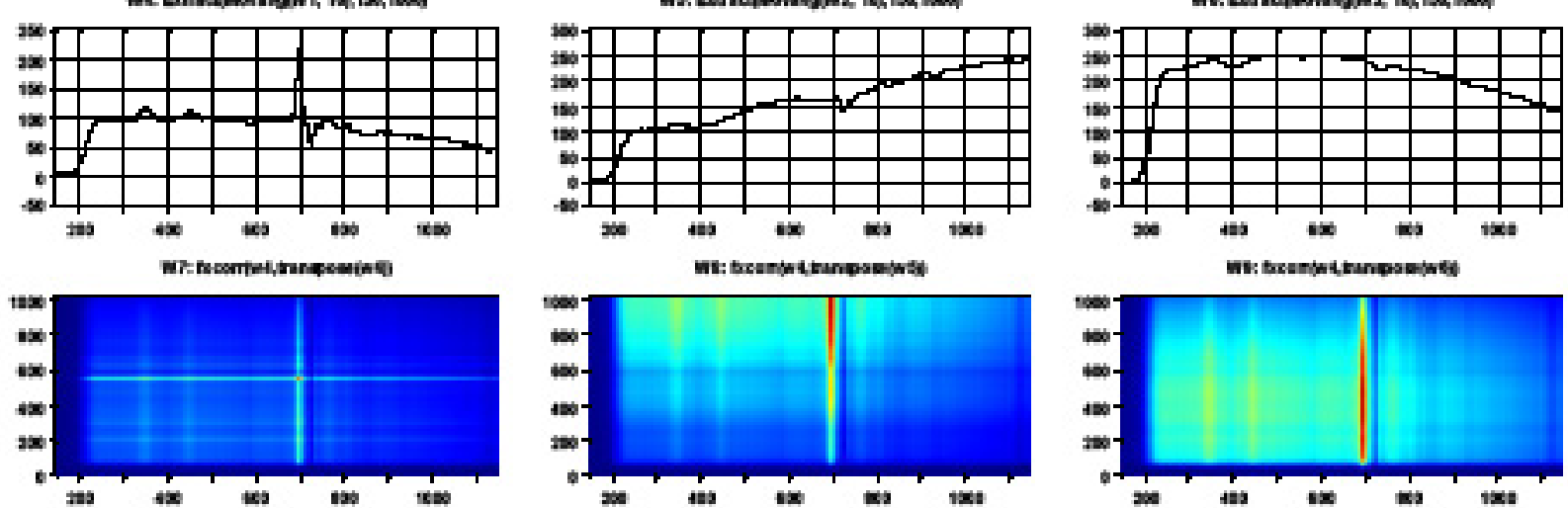

Figure 7: Raw data Raman spectra are not corrected of sound and carious tooth are illustrated in window 1, window 2 and window 3, respectively as in Fig. 3. The decayed teeth are classified into two classes, moderate (window 3 ) and severed carious (window 2). In windows 4, 5 and 6, the collected Raman spectra after data noise reduction using 10 moving average filter and normalization. 2D cross-correlationof sound enamel (w7), moderate carious (w9) and severed carious enamel (w8).

ten statistically different peaks in the region of 200 the $1300 \mathrm{~cm}^{-1}$ as shown in Figure 7.

Figure 7 shows a typical example of a synchronous 2D correlation spectrum plotted as a contour map. A synchronous spectrum references about the corresponding molecular structure of this vibration symmetric spectrum with respect to a diagonal line corresponding to spectral coordinates (Raman spectrum of sound teeth at position $1=$ Raman spectrum of sound teeth at position 2). Correlation peaks appear at both diagonal positions. The intensity of peaks located at diagonal positions corresponds to the autocorrelation function of spectral intensity variations observed during a period T. The diagonal Peaks are therefore referred to as autopeaks. In the Raman spectrum of sound teeth, there is one auto peaks located at the spectral coordinates 953.87. The intensity of autopeaks of a synchronous correlation spectrum, which is always positive, represents the overall extent of dynamic fluctuations of spectral intensity with respect to the intensity of the reference spectrum observed at the specific wave number during the period $\mathrm{T}$. Thus, regions of a dynamic spectrum which change intensity to a greater extent will show stronger autopeaks, while those remaining constant give little or no autopeaks.

Asynchronous cross peak develops only if the intensities of two dynamic spectral intensities vary out of phase as shown in Figures 5 (a), (b) (Raman spectrum of reference sound teeth and Raman spectrum of carious teeth detection).

This feature is especially useful in differentiating and diagnosing overlapped bands arising from different spectral origins or moieties. Thus, spectral intensity contributions from different components of a mixture, from materials indifferent phases or from chemical functional groups experiencing different effects due to some external fields could be discriminated as in carious Raman spectrum. As 
long as the temporal signatures or the patterns of time-dependent variation of spectral intensities are substantially different, asynchronous cross peaks will develop among them. The appearance of asynchronous cross peaks indicates that these bands arise from different molecular vibrations.

The sign of an asynchronous cross peak becomes positive if the intensity changes at Raman spectrum of reference sound teeth and Raman spectrum occurs predominantly before Raman spectrum of carious teeth. It becomes negative, on the other hand, if the change occurs after Raman spectrum of carious teeth.

Figure 5 indicates that the intensity changes (either an increase or a decrease) at bands Raman spectrum of carious teeth after the reference one. So far, we have demonstrated that carious enamel can be distinguished from sound enamel at micro- spectroscopic level using the intensity changes of the hydroxyapatite Raman bands. We proceeded to investigate the suitability of a fiber optic Raman probe and 2D cross-correlation algorithm for caries detection. A more complete understanding of the relationship between different modes and the integrated intensities can be gained by examining the intensity changes across the entire experimental data set. The intensity of peaks located at diagonal positions corresponds to the autocorrelation function of spectral intensity variations observed during a period $\mathrm{T}$ shifted from the center as carious decayed increased as shown in Figure 5(a).

These changes in integrated peak intensity of the full data set of Raman spectra for unknown teeth can be examined via 2D correlation analysis as discussed before. If the modes are fluctuating in a truly independent fashion, then the time evolution of the four Raman modes should be non-correlated.

Figure 8 shows two teeth (E1, E2) trained to use the $2 \mathrm{D}$ correlation analysis. The examined 2D correlation analysis is a powerful tool of carious detection. If the examined tooth is decayed, the Raman modes will be anti- correlated at the carious position as shown in figure indicating that changes in the vibrational features are not inversely related. This non-correlation of the data implies that the modes themselves are fluctuating in a truly independent fashion. The scanned normal teeth Raman spectra demonstrated that a strong correlation exists between the Stokes bands and all the vibrational modes after the spectra were normalized prior to analysis in an attempt to compensate for the changes in total intensity. There is no anti-correlation presence between the modes in the spectrum.

This apparently surprising result may be readily explained by the fact that the dynamic spectra shown in Figures 4 and 7 are chosen in such a way that they represent responses by an identical system to two very different spectrums giving us the response transfer function of the system as calculated and illustrated in Table 1. The severedd carious teeth spectrum shown in figure response with respect to sound one are 0.1033 , while the calculated response of moderate carious is 0.3202 .

Table 1: Examiner agreement and reliability when assessing sound versus any demineralization and carious

\begin{tabular}{cc} 
Examined Teeth & $\begin{array}{c}\text { Correlation } \\
\text { Coefficient }\end{array}$ \\
\hline Class a (Sound teeth) & 0.978 \\
\hline Class b (moderate decayed teeth) & 0.3202 \\
\hline Class b (severed decayed teeth) & 0.1033
\end{tabular}

\section{Conclusion}

In this study, we explored the potential of using Raman spectroscopy for the early detection and characterization of enamel carious and demonstrated the detailed information that could be obtained from Raman spectra 2D cross-correlation algorithm; this technique has the potential to provide both qualitative and quantitative diagnostic information in a non-destructive and timely fashion. This in- 

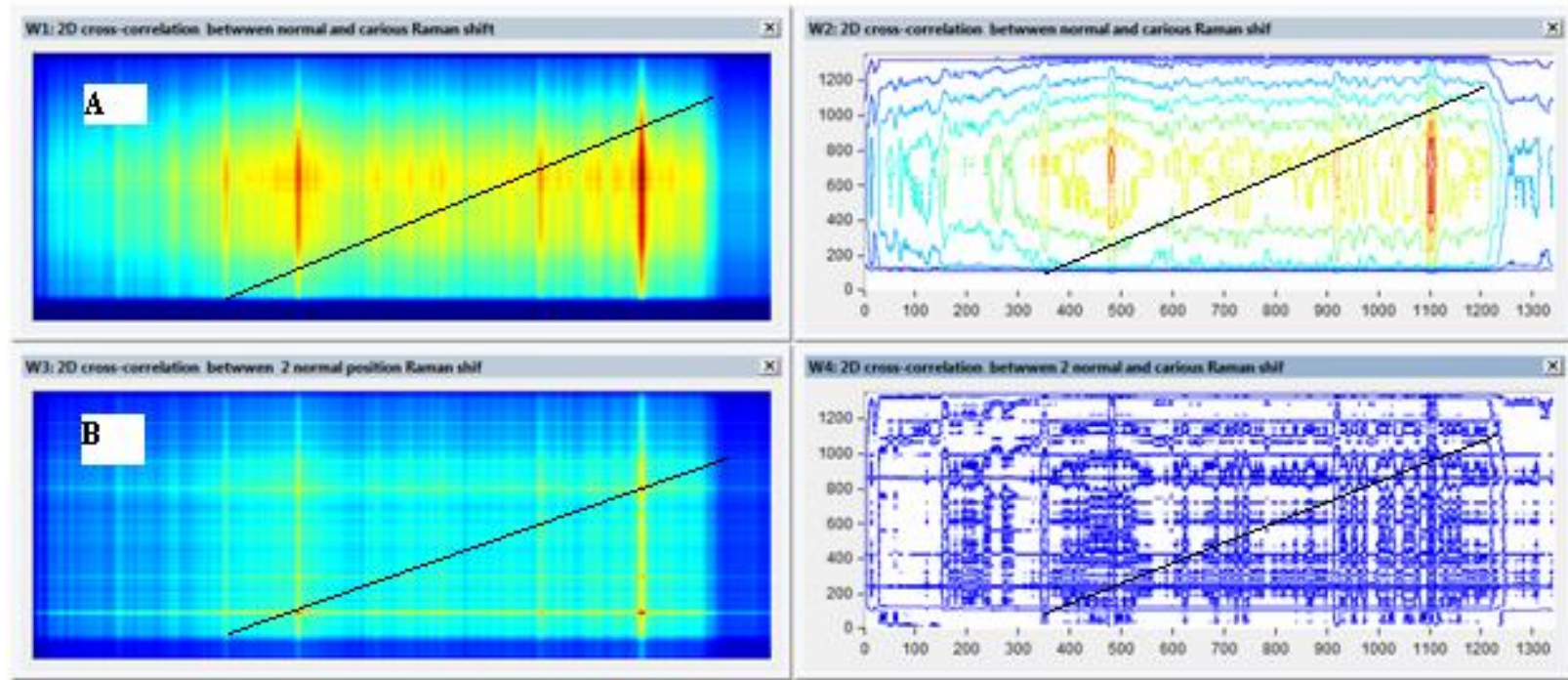

Figure 8: Shows a typical unknown human teeth examination using Raman spectrum 2D correlation Algorithm (A) carious teeth detection plotted as a density and contour map, (B) sound teeth detection plotted as a density and contour map.

formation might be used both for diagnostic screening and the study of disease progression so that optimal treatment methods may be developed and monitored. A generalized two-dimensional correlation method for IR, Raman, and other types of spectroscopy is introduced. The new formalism is applicable to complex time-dependent spectral intensity variations (dynamic spectrum) induced by Raman spectra response of sound and decayed teeth. A set of 2D correlation spectra is generated from such a dynamic spectrum. The synchronous 2D correlation spectrum represents coupled or related changes of spectral intensity variations, while asynchronous correlation and disrelation spectra represent independent or separate variations. Potential use of $2 \mathrm{D}$ correlation spectroscopy is now expanded to a very wide range of applications.

\section{Conflict of Interest}

\section{None}

\section{References}

1. Kopycka-Kedzierawski DT, Billings RJ, McConnochie KM. Dental screening of preschool children using teledentistry: a feasibility study. Pediatr Dent. 2007;29:209-13. PubMed PMID: 17688017.
2. Kopycka-Kedzierawski DT, Bell CH, Billings RJ. Prevalence of dental caries in Early Head Start children as diagnosed using teledentistry. Pediatr Dent. 2008;30:329-33. PubMed PMID: 18767513.

3. Angmar-Mansson B, ten Bosch JJ. Optical methods for the detection and quantification of caries. Adv Dent Res. 1987;1:14-20. PubMed PMID: 3481546.

4. In: ASTM International. Standard Guide for Raman Shift Standards for Spectrometer Calibration. Active Standard ASTM E1840. Available from: http://www.astm.org/Standards/E1840.htm.

5. Baraga JJ, Rava RP, Manoharan R, Feld MS, Kramer JR. Vibrational spectmscopy: new techniques for in situ optical analysis of human tissue. In: Photodynarnic Thempy and Biomedics Lasers. Spinelli P, M. Dal Fante M, Marchd R, eds. New York: Elsevier Science Publishing; 1992. p. 65-73.

6. Hintze H. Screening with conventional and digital bite-wing radiography compared to clinical examination alone for caries detection in lowrisk children. Caries Res. 1993;27:499-504. doi.org/10.1159/000261588. PubMed PMID: 8281566.

7. Pitts NB. Diagnostic tools and measurements--impact on appropriate care. Community Dent Oral Epidemiol. 1997;25:24-35. doi. org/10.1111/j.1600-0528.1997.tb00896.x. PubMed PMID: 9088689. 
8. Verdonschot EH, Angmar-Mansson B, ten Bosch JJ, Deery CH, Huysmans MC, Pitts NB, et al. Developments in caries diagnosis and their relationship to treatment decisions and quality of care. ORCA Saturday Afternoon Symposium 1997. Caries Res. 1999;33:32-40. doi.org/10.1159/000016493. PubMed PMID: 9831778.

9. Bader JD, Shugars DA. The evidence supporting alternative management strategies for early occlusal caries and suspected occlusal dentinal caries. J Evid Based Dent Pract. 2006;6:91-100. doi.org/10.1016/j.jebdp.2005.12.004. PubMed PMID: 17138407.

10. Angmar-Mansson B, ten Bosch JJ. Advances in methods for diagnosing coronal caries--a review. Adv Dent Res. 1993;7:70-9. PubMed PMID: 8260014.

11. Passarelli Jr RE, Siggia AD. The autocorrelation function and Doppler spectral moments: Geometric and asymptotic interpretations. Journal of cli- mate and applied meteorology. 1983;22:1776-87. doi.org/10.1175/1520-0450(1983)022<1776:TAFA DS>2.0.C0;2.

12. Kay SM. Fundamentals of statistical signal processing, volume I: estimation theory. 1993.

13. Janssen LH, Van Der Spek GA. The shape of Doppler spectra from precipitation. Aerospace and Electronic Systems, IEEE Transactions on. 1985;(2):208-19.

14. Wei M, Evans JH, Bostrom T, Grondahl L. Synthesis and characterization of hydroxyapatite, fluoride-substituted hydroxyapatite and fluorapatite. J Mater Sci Mater Med. 2003;14:311-20. doi. org/10.1023/A:1022975730730. PubMed PMID: 15348455.

15. Campillo M, Lacharmoise PD, Reparaz JS, Goni AR, Valiente M. On the assessment of hydroxyapatite fluoridation by means of Raman scattering. J Chem Phys. 2010;132:244501. doi.org/10.1063/1.3428556. PubMed PMID: 20590200. 